\title{
The hindered rotor density-of-states interpolation function
}

\author{
Richard B. McClurg and Richard C. Flagan \\ Spalding Laboratory (210-41), Division of Chemistry and Chemical Engineering, California Institute \\ of Technology, Pasadena, California 91125 \\ William A. Goddard III ${ }^{\text {a) }}$ \\ Materials and Process Simulation Center, Beckman Institute (139-74), Division of Chemistry and Chemical \\ Engineering, California Institute of Technology, Pasadena, California 91125
}

(Received 30 October 1996; accepted 17 January 1997)

\begin{abstract}
We construct an approximation to the partition function for hindered rotors based entirely on their asymptotic behavior and no fitting parameters. The approximant is shown to be quite accurate in all temperature ranges. Explicit auxiliary functions are derived for the Helmholtz free energy, internal energy, heat capacity, and entropy. We apply this function to estimating the heat capacity and unimolecular dissociation rate for ethane. () 1997 American Institute of Physics.
\end{abstract}

[S0021-9606(97)50816-6]

\section{INTRODUCTION}

Since the pioneering work of Pitzer ${ }^{1,2}$ which showed that torsions about single bonds are not freely rotating, there have been many books ${ }^{3-5}$ and reviews ${ }^{6-11}$ on hindered rotation. The quantum mechanics of hindered rotors is well known, but the theory has been too cumbersome for many applications. Calculating the values from first principles requires evaluating and diagonalizing a large matrix. This is practical with modern computers, but this is often an unwelcomed diversion in the context of a larger problem. Pitzer's published tables for the thermodynamic functions of hindered rotors $^{1}$ are also inconvenient for use in computer programs. We would like to have a function that estimates the thermodynamic functions for hindered rotors without having to start from first principles for each application. One such function was proposed by Truhlar. ${ }^{12}$ We will refer to this function as T91 hereafter. Herein we present an improvement, the Hindered Rotor Density-of-States (HRDS) interpolation function, which is asymptotically correct at low and high temperatures and accurate at intermediate temperatures.

In Secs. II and III, we derive asymptotic forms for the low-temperature (quantum) and high-temperature (classical) canonical partition functions $(Q)$. The reader interested in applying the methods may skip to Sec. IV which presents the HRDS interpolation function based on the asymptotic behavior of the partition function. There are no fitting parameters in determining this function. Instead, we construct a product of terms determined such that the function has the correct asymptotic behavior at both low and high temperature. By comparing with numerical values, we show that the function is accurate over the entire temperature range, much better than the previous function. ${ }^{12}$ In Sec. V, we compute thermodynamic functions based on the HRDS function. Finally, Sec. VI gives two applications: calculation of the gas phase heat capacity of ethane and the dissociation rate constant of

\footnotetext{
a) Author to whom correspondence should be addressed; electronic mail: wag@wag.caltech.edu
}

ethane. These examples serve to illustrate the benefits of the new function.

\section{LOW-TEMPERATURE (QUANTUM) LIMIT}

In the low-temperature limit, the partition function must be calculated using the discrete energy levels computed using quantum mechanics. The time-independent Schrödinger equation for rotation about a single axis is ${ }^{13}$

$$
-\frac{\hbar^{2}}{2 I} \frac{d^{2} \Psi}{d \phi^{2}}+V \Psi=E \Psi, \quad 0 \leqslant \phi<2 \pi .
$$

Here, $I$ is the reduced moment of inertia about the axis parametrized by $\phi$. In many cases, $I$ changes only slightly with $\phi$ and can be treated as a constant. Also, the potential $(V)$ can be approximated using

$$
V=\frac{W}{2}(1-\cos n \phi),
$$

where $W$ is the height of the potential barrier, $n$ is the number of equivalent minima, and $\phi=0$ corresponds to a minimum.

With the following definitions,

$$
\begin{aligned}
& 2 x=n \phi, \\
& \alpha=\frac{8 I}{\hbar^{2} n^{2}}(E-W / 2), \\
& 2 r^{2}=\frac{8 I}{\hbar^{2} n^{2}}(W / 2) .
\end{aligned}
$$

Equation (1) transforms into Mathieu's equation: ${ }^{14,15}$

$$
\frac{d^{2} \Psi}{d x^{2}}+\left(\alpha+2 r^{2} \cos 2 x\right) \Psi=0, \quad 0 \leqslant x<n \pi .
$$

As discussed by Nielsen, ${ }^{16}$ the periodicity of $n \pi$ in the potential requires solutions to Eq. (6) having period $n \pi$, which have been studied primarily for $n=1,2$. Equation (6) has solutions only for characteristic values $(\alpha)$ which depend on $r$ and $n$. 


\section{A. Large barrier limit (harmonic oscillator)}

For barrier heights $(W)$ which are large compared to both $E$ and $\hbar \omega, r$ is large and only the region of $x$ near 0 is significant. Expanding Eq. (6) for small $x$ leads to a perturbed harmonic oscillator (HO) with characteristic values $(\alpha)$

$$
\alpha=-2 r^{2}+(2+4 m) r-\frac{1}{4}\left(1+2 m+2 m^{2}\right)+\mathrm{O}\left(m^{3} / r\right)
$$

$m=0,1,2, \ldots$

that are $n$-fold degenerate, but otherwise independent of the periodicity $(n) .{ }^{15}$ Here, $m$ is an integer index for the characteristic values. Solving Eqs. (4), (5), and (7) for $E$ yields

$$
\begin{aligned}
E_{m}= & \frac{\hbar^{2} n^{2} r}{2 I}\left[(1 / 2+m)-\frac{1}{16 r}\left(1+2 m+2 m^{2}\right)\right. \\
& \left.+\mathrm{O}\left(m^{3} / r^{2}\right)\right] .
\end{aligned}
$$

Defining the HO frequency $(\omega)$ as

$$
\omega=n \sqrt{\frac{W}{2 I}},
$$

we see that Eq. (8) reduces to the energy levels of a harmonic oscillator ${ }^{13}$ as $r$ approaches $\infty$,

$$
\begin{aligned}
& E_{m}=E_{m}^{\mathrm{HO}}-\frac{\hbar \omega}{16 r}\left(1+2 m+2 m^{2}\right)+\mathrm{O}\left(m^{3} / r^{2}\right), \\
& E_{m}^{\mathrm{HO}}=\hbar \omega(1 / 2+m) .
\end{aligned}
$$

The first effect of finite $r$ is to lower the allowed energies relative to the corresponding $\mathrm{HO}$ values.

Eliminating $I$ from Eqs. (5) and (9) yields

$$
r=W / \hbar \omega \text {. }
$$

Therefore, $r$ is the ratio of the barrier height to the harmonic oscillator frequency (which is based on the curvature at the bottom of the potential well). Equivalently, $r$ is a rough estimate of the number of quantum levels classically confined within a single well.

Using the limiting form for $E$ at small $m$, we can calculate a limiting form for the canonical partition function $(Q)$ at low temperature,

$$
Q=\sum_{m} \exp \left(-E_{m} / k T\right) .
$$

At sufficiently low temperature, $Q$ is dominated by the first few terms of the summation in Eq. (13), for which the harmonic oscillator limit [Eq. (10)] holds,

$$
\begin{aligned}
& \lim _{T \rightarrow 0} Q=\sum_{m=0}^{\infty} \exp \left[\frac{-E_{m}^{\mathrm{HO}}+\frac{\hbar \omega}{16 r}\left(1+2 m+2 m^{2}\right)}{k T}\right], \\
& Q^{\mathrm{HO}}=\sum_{m=0}^{\infty} \exp \left[-E_{m}^{\mathrm{HO}} / k T\right]=\frac{\operatorname{csch}[\hbar \omega / 2 k T]}{2} .
\end{aligned}
$$

Taking the first two terms of each summation and expanding yields:

$\lim _{T \rightarrow 0} \frac{Q}{Q^{\mathrm{HO}}}=\exp \left[\frac{\hbar \omega}{16 r k T}\right]\left(1+\exp \left[\Delta+\frac{\hbar \omega}{4 r k T}\right]-\exp [\Delta]\right)$,

$\Delta=\left(E_{0}^{\mathrm{HO}}-E_{1}^{\mathrm{HO}}\right) / k T=-\hbar \omega / k T$.

Defining a non-dimensional temperature $(\Theta)$,

$$
\Theta=k T / \hbar \omega,
$$

simplifies Eq. (16) and helps quantify "sufficiently low temperature,"

$$
\begin{aligned}
\lim _{\Theta \ll 1} \frac{Q}{Q^{\mathrm{HO}}}= & \exp \left[\frac{1}{16 r \Theta}\right]\left(1+\exp \left[\frac{-1}{\Theta}+\frac{1}{4 r \Theta}\right]\right. \\
& \left.-\exp \left[\frac{-1}{\Theta}\right]\right) .
\end{aligned}
$$

Thus, for any finite value of $r$, the actual partition function exceeds $Q_{\mathrm{HO}}$ at low temperature. This follows directly from Eq. (11) since the first effects of finite $r$ are to lower the allowed energies relative to the corresponding harmonic oscillator levels. The second term in Eq. (18) cannot be expanded about $\Theta=0$. (The origin is an essential singular point $^{17}$ of $\exp [ \pm \alpha / \Theta]$.) Therefore, we use the hightemperature asymptote.

\section{B. Small barrier limit (free rotor)}

If the barrier height $(W)$ is small compared to $E$, then solutions to Eq. (6) are sums of slightly distorted sines and cosines with characteristic values ${ }^{14,15}$

$$
\alpha=\left(\frac{2 m}{n}\right)^{2}+\frac{r^{4}}{2\left[(2 m / n)^{2}-1\right]}+\mathrm{O}\left(r^{6} / m^{4}\right) .
$$

Solving Eqs. (4) and (19) for $E$ yields

$$
\begin{aligned}
& E_{m}=\frac{W}{2}+\frac{m^{2} \hbar^{2}}{2 I}+\frac{r^{4} \hbar^{2} n^{2}}{16 I\left[(2 m / n)^{2}-1\right]}+\mathrm{O}\left(r^{6} / m^{4}\right) \\
& m=0, \pm 1, \pm 2, \ldots .
\end{aligned}
$$

The first two terms in Eq. (20) correspond to the energy levels of a one-dimensional free rotor $^{13}$ displaced by the average potential energy $(W / 2)$. This series is accurate as $m$ approaches $\infty$, but is not uniformly convergent. The second term in Eq. (19) is correct for $|m|>n / 2$. For smaller values of $m$, there are expansions analogous to Eq. (19), but those expansions must be derived individually for each (small) value of $m$. Higher-order terms in Eq. (19) are also correct for large $|m|$, but they similarly break down for small values of $m$. The truncated series is not accurate for $|m|<r n / 2$. For these reasons, we use a different approach for the hightemperature limit in Sec. III.

\section{HIGH-TEMPERATURE (CLASSICAL) LIMIT}

At high temperatures, the canonical partition function $(Q)$ has significant contributions from a large number of quantum states. See Eq. (13). In this limit, it is useful to use 
classical statistical mechanics in which the summation over states is approximated as an integral over phase space ${ }^{1}$

$$
\lim _{T \rightarrow \infty} Q=\frac{1}{h} \int_{-\infty}^{\infty} \int_{\alpha}^{\beta} \exp \left[\frac{-\mathscr{H}}{k T}\right] d q d p .
$$

Here, $\alpha$ and $\beta$ are limits on the spatial coordinate $(q), \mathscr{H}$ is the Hamiltonian, and $p$ is the momentum. For a rigid rotor with hindering potential $V(\phi)$, this reduces to

$$
\lim _{T \rightarrow \infty} Q=\frac{1}{n h} \int_{-\infty}^{\infty} \exp \left[\frac{-J^{2}}{2 I k T}\right] d J \int_{0}^{2 \pi} \exp \left[\frac{-V}{k T}\right] d \phi,
$$

where $J$ is the angular momentum. The factor $1 / n$ eliminates over-counting of states due to the $n$ equivalent wells. For the cosine potential in Eq. (2), the second integral can be simplified,

$$
\begin{aligned}
\lim _{T \rightarrow \infty} Q= & \frac{2 \exp [-W / 2 k T]}{n h} \int_{-\infty}^{\infty} \exp \left[\frac{-J^{2}}{2 I k T}\right] d J \\
& \times \int_{0}^{\pi} \exp \left[\frac{W}{2 k T} \cos \phi\right] d \phi .
\end{aligned}
$$

Both of these integrals are in standard forms, ${ }^{18}$ which can be integrated to give

$$
\lim _{T \rightarrow \infty} Q=\left\{\frac{(2 \pi I k T)^{1 / 2}}{n \hbar}\right\} \exp \left[\frac{-W}{2 k T}\right] \mathrm{I}_{0}\left[\frac{W}{2 k T}\right] .
$$

The first term in Eq. (24) is the classical partition function for a one-dimensional free rotor $\left(Q^{\mathrm{FR}}\right) .{ }^{13}$ The second term is a Boltzmann factor for the average potential energy. The third term, $\mathrm{I}_{0}$, is a zeroth-order modified Bessel function, ${ }^{15,18}$ which accounts for the rotor preferentially residing in the potential wells (as opposed to the potential maxima) at finite temperature. An equivalent function was used by Pitzer and Gwinn. $^{2}$ Using the notation of Sec. II, Eq. (24) can be simplified

$$
\lim _{\Theta \gg 1} Q=(\pi \Theta r)^{1 / 2} \exp \left[\frac{-r}{2 \Theta}\right] \mathrm{I}_{0}\left[\frac{r}{2 \Theta}\right] .
$$

This asymptote is depicted in Fig. 1 along with numerical results, both for $r=3$. The numerical results were obtained by solving Eq. (6) for the first 400 characteristic values $(\alpha)$ using an eigenfunction expansion. ${ }^{19,20}$ The numerical results agree with tabulated values. ${ }^{14}$ We then calculated $Q$ using Eq. (13) directly. In Fig. 1, we see that $Q$ approaches the low temperature limit for $\Theta \ll 1$ and the high temperature limit for $\Theta \gg 1$.

Since Eq. (15) is the partition function for a harmonic oscillator at any temperature, it can be expanded in the limit of high temperature to give the classical limit for $Q^{\mathrm{HO}}$,

$$
\lim _{\Theta \gg 1} Q^{\mathrm{HO}}=\Theta+\mathrm{O}\left(\Theta^{-1}\right) .
$$

We use this result in Sec. IV.

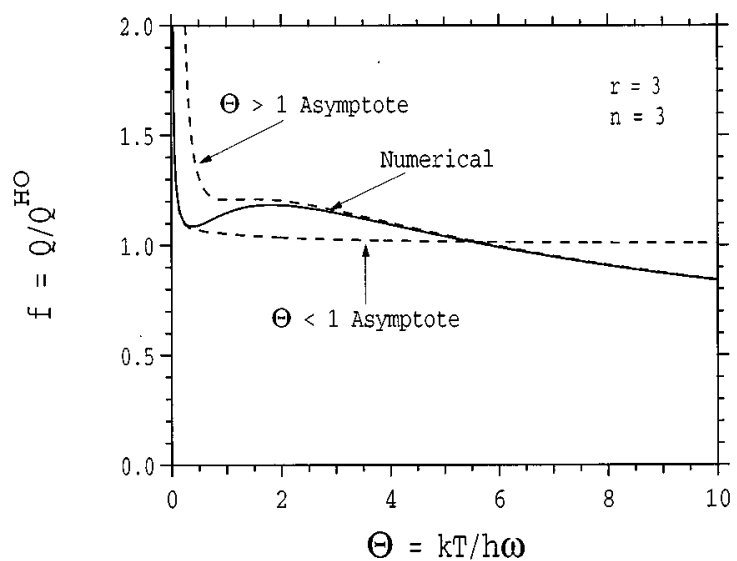

FIG. 1. Asymptotes for $\Theta \ll 1$ (Eq. 18) and $\Theta \gg 1$ (Eq. 25). For $\Theta \approx 2 r / 3$, the hindered rotor is intermediate between a harmonic oscillator and a free rotor, requiring the use of an interpolation function.

\section{THE HINDERED ROTOR DENSITY-OF-STATES (HRDS) FUNCTION}

In this section, we construct an interpolation function having the asymptotic forms determined in Secs. II and III. For this purpose, it is convenient to define $f$ as follows:

$$
f=Q / Q^{\mathrm{HO}} \text {. }
$$

Then Eqs. (18), (25), and (26) provide limiting values for $f$

$$
\begin{aligned}
& \lim _{\Theta \ll 1} f=\exp \left[\frac{1}{16 r \Theta}\right](1+\mathrm{O}(\exp [-\alpha / \Theta])), \\
& \lim _{\Theta \gg 1} f=\left(\frac{\pi r}{\Theta}\right)^{1 / 2} \exp \left[\frac{-r}{2 \Theta}\right] \mathrm{I}_{0}\left[\frac{r}{2 \Theta}\right] .
\end{aligned}
$$

The first term in Eq. (28) comes from the zero-point energy which is a quantum-mechanical effect. The remainder of Eq. (28) and all of Eq. (29) are from density of states considerations. Therefore, we write Eq. (27) in the following form:

$$
f=P \exp \left[\Delta E^{z p} / k T\right] .
$$

We use the following Padé approximant for $\Delta E^{z p}$

$$
\Delta E^{z p}=\frac{\hbar \omega}{2+16 r}
$$

and approximate $P$ using its high-temperature limit

$$
P=\left(\frac{\pi r}{\Theta}\right)^{1 / 2} \exp \left[\frac{-r}{2 \Theta}\right] \mathrm{I}_{0}\left[\frac{r}{2 \Theta}\right] .
$$

The Padé approximant corrects for the over-estimate of the zero-point energy in the HO reference function. For large $r$, the over-estimate is $\hbar \omega / 16 r$. See Eq. (10). For $r=0$, there is no hindering potential, so $E^{z p}=0$ and the over-estimate is $\hbar \omega / 2$. Using the following expansion for $\mathrm{I}_{0}[z]$ where $z$ can be complex ${ }^{15,18}$

$$
\lim _{|z| \rightarrow \infty} \mathrm{I}_{0}[z]=\exp [z] /(2 \pi z)^{1 / 2},
$$




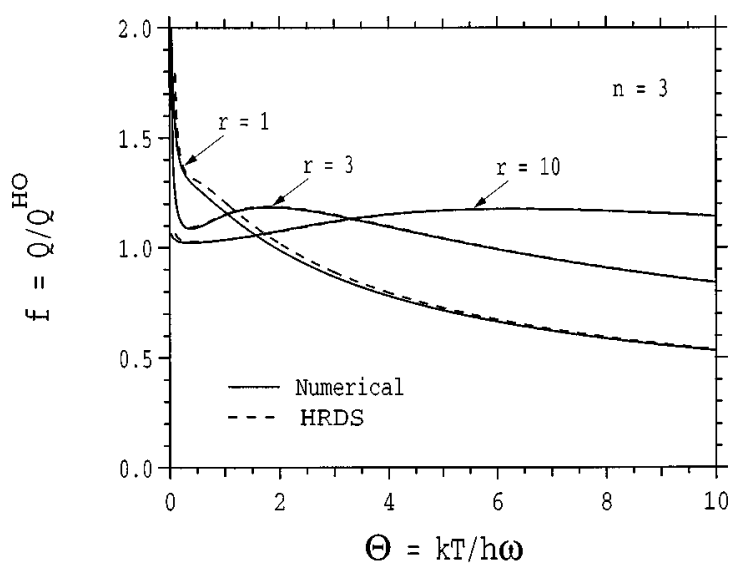

FIG. 2. Accuracy of the HRDS interpolation function for various values of $r$. This shows that HRDS is accurate for both mildly hindered rotors $(r \approx 1)$ and strongly hindered rotors $(r>1)$. On the scale of this plot, the curves are indistinguishable for $r$ greater than about two.

it is simple to confirm that $P$ approaches unity at low temperatures. We refer to $P$ as the Hindered Rotor Density-ofStates (HRDS) interpolation function. In Fig. 2, we show that the HRDS function is accurate for both mildly hindered rotors $(r \approx 1)$ and strongly hindered rotors $(r>1)$. This is significantly more accurate than the previous interpolating function, T91, as shown in Fig. 3. The greatest improvement is in the neighborhood of $\Theta=2 r / 3$ where the hindered rotator is intermediate between a harmonic oscillator and a free rotor.

\section{THERMODYNAMIC FUNCTIONS}

Using our estimate for $f$ [Eqs. (30) and (32)], we calculate $^{13}$ deviations from the harmonic oscillator value for the Helmholtz free energy $(\Delta A)$,

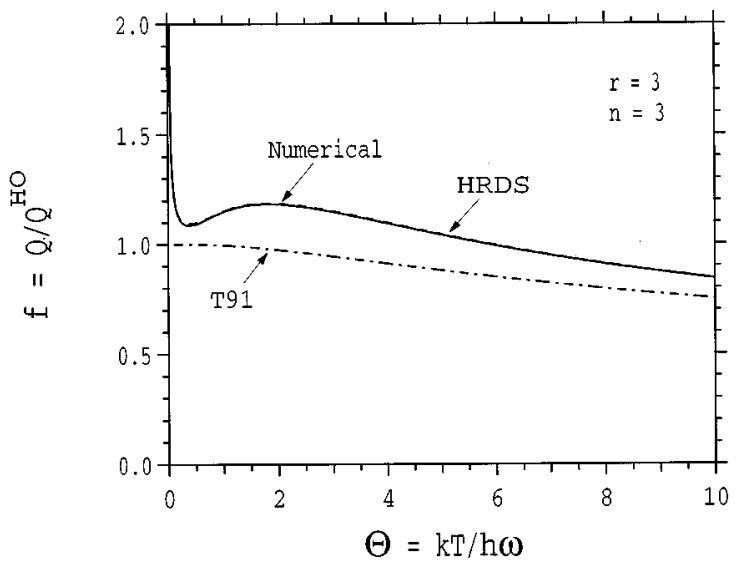

FIG. 3. Accuracy of the current work (HRDS) and an alternative interpolation scheme by Truhlar (T91) (Ref. 12). The current work better represents the numerical results, especially near $\Theta=2 r / 3$ where the hindered rotor is intermediate between the harmonic oscillator limit and the free rotor limit.

$$
\begin{aligned}
\Delta A / k T & =-\ln f \\
& =\frac{-1}{(2+16 r) \Theta}+\frac{r}{2 \Theta}-\ln \left\{\left(\frac{\pi r}{\Theta}\right)^{1 / 2} \mathrm{I}_{0}[r / 2 \Theta]\right\} ;
\end{aligned}
$$

internal energy $(\Delta U)$,

$$
\begin{aligned}
\Delta U / k T & =T \frac{d(\ln f)}{d T} \\
& =\frac{-1}{2}-\frac{1}{(2+16 r) \Theta}+\frac{r}{2 \Theta}\left\{1+\frac{\mathrm{I}_{1}[r / 2 \Theta]}{\mathrm{I}_{0}[r / 2 \Theta]}\right\} ;
\end{aligned}
$$

heat capacity $\left(\Delta C_{v}\right)$,

$$
\begin{aligned}
\Delta C_{v} / k & =T \frac{d^{2}(T \ln f)}{d T^{2}} \\
& =\frac{-1}{2}+\frac{r^{2}}{8 \Theta^{2}}\left\{1-2\left(\frac{\mathrm{I}_{1}[r / 2 \Theta]}{\mathrm{I}_{0}[r / 2 \Theta]}\right)^{2}+\frac{\mathrm{I}_{2}[r / 2 \Theta]}{\mathrm{I}_{0}[r / 2 \Theta]}\right\} ;
\end{aligned}
$$

and entropy $(\Delta S)$,

$$
\Delta S / k=\Delta U / k T-\Delta A / k T .
$$

There are widely available routines for calculating $\mathrm{I}_{\nu}[x]$ where $x$ is real and $\nu$ is an integer. ${ }^{21}$ Alternatively, the following expressions can be used: ${ }^{15,18}$

$$
\begin{aligned}
& \mathrm{I}_{\nu}[x]=\sum_{k=0}^{\infty} \frac{(x / 2)^{\nu+2 k}}{k !(k+\nu) !}, \\
& \mathrm{I}_{\nu}[x]=\frac{\exp [x]}{(2 \pi x)^{1 / 2}} \sum_{k=0}^{\infty} \frac{(-1)^{k} \Gamma[\nu+k+1 / 2]}{(2 x)^{k} k ! \Gamma[\nu-k+1 / 2]} .
\end{aligned}
$$

The first expression is recommended ${ }^{15}$ for $|x|<3.75$ and the second for $|x|>3.75$.

\section{APPLICATIONS}

The only input parameters to HRDS are the periodicity $(n)$, the ratio of barrier height to harmonic frequency $(r)$, and the scaled temperature $(\Theta)$. Before applying HRDS to calculate the heat capacity and rate constant for dissociation of ethane, we briefly discuss how to estimate these values.

For simple cases, $n$ can be determined by symmetry. Following the notation of Wolfe, ${ }^{22}$ consider a torsion between atoms $M$ and $N$ with ligands $a, b, c, x, y$, and $z$. The ligands can be atoms, groups of atoms, or lone pairs of electrons.

$a b c M-N x y z$ : If $a, b$, and $c$ (and/or $x, y$, and $z$ ) are identical, then there are three equivalent minima. $(n=3)$ (e.g. Ethane $\left\{\mathrm{H}_{3} \mathrm{C}-\mathrm{CH}_{3}\right\}$, Methyl amine $\left\{\mathrm{H}_{2} \mathrm{~N}-\mathrm{CH}_{3}\right\}$, and Methanol $\left.\left\{\mathrm{HO}-\mathrm{CH}_{3}\right\}\right)$

$a b c M-N x y$ : If $a, b$, and $c$ are identical and $x$ and $y$ are identical, then there are six equivalent minima. $(n=6)$ (e.g. Methyldifluoroborane $\left\{\mathrm{CH}_{3}-\mathrm{BF}_{2}\right\}$ and Toluene $\left\{\mathrm{H}_{3} \mathrm{C}-\mathrm{Ph}\right\}$ where $\mathrm{N}, \mathrm{x}$, and $y$ are all part of the phenyl ring)

(iii) $a b c M-N x y z$ : For particular choices of ligands, 


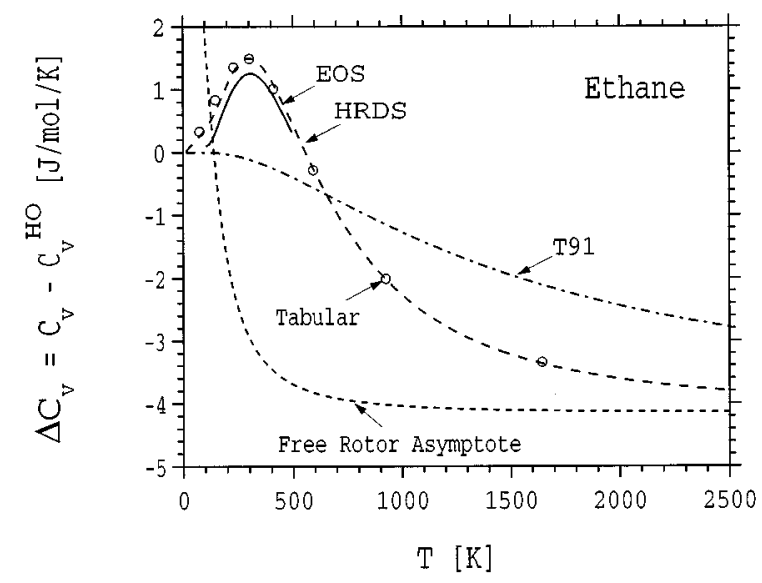

FIG. 4. Comparison of the deviations from the harmonic oscillator heat capacity for ethane as calculated using linear interpolation from Pitzer's tables (Tabular) (Ref. 2), the T91 interpolation function (Ref. 12), and the current work (HRDS). The equation of states curve (EOS) is the result of fitting a partition function to a critically analyzed set of experimental data (Ref. 24). Also shown is the rigid rotor (high-temperature) asymptote. HRDS agrees well with the tabulated results and the EOS.

there may be two equivalent minima. $(n=2)$ (e.g., HSSH has two minima at $\phi= \pm 90 \mathrm{deg}$.)

For torsions that do not meet the restrictions of the above three cases, the potential may not be well-represented by Eq. (2) and this method may not be appropriate. For example, Hydrogen peroxide $(\mathrm{HO}-\mathrm{OH})$ has two equivalent minima separated by one large and one small barrier. ${ }^{22}$ Diphenyl $(\mathrm{Ph}-\mathrm{Ph})$ has four minima with alternating large and small barriers to rotation. These potentials cannot be represented using a single Fourier component as in Eq. (2).

The harmonic oscillator frequency $(\omega)$ and the height of the potential barrier $(W)$ can be measured experimentally or calculated using quantum mechanics or empirical force fields. See Berg ${ }^{11}$ for a recent survey of the various methods. Also, see Lowe ${ }^{8}$ for an extensive tabulation of experimental data. Once $\omega$ and $W$ are known and the temperature of interest (T) is chosen, $r$ and $\Theta$ are calculated using Eqs. (12) and (17).

\section{A. Ethane heat capacity}

As a sample application of the HRDS function, we calculate the heat capacity of ethane. Heat capacity provides a stringent test of the shape of the partition function since it requires second derivatives. See Eq. (36). We have used spectroscopic data recommended by $\mathrm{Chao}^{23}\left(\omega=289 \mathrm{~cm}^{-1}\right.$, $W=2.96 \mathrm{kcal} / \mathrm{mol}, n=3$ ). For comparison with experimental data, we use an equation of state for ethane based on a critical review of the available spectroscopic and thermodynamic experiments. $^{24}$

Figure 4 shows that the HRDS interpolation function provides a good approximation to the experimental data in the temperature range where data is available. Both HRDS and T91 utilize the harmonic oscillator and rigid rotor limiting behavior, but HRDS puts greater emphasis on accurately reflecting how those asymptotes are reached. This is particu- larly evident in the neighborhood of the peak in $\Delta C_{v}$ where the hindered rotor is far from either limiting case. For ethane, this occurs near room temperature.

\section{B. Ethane dissociation rate constant}

Our second illustration is estimating the high-pressure, unimolecular rate constant for ethane dissociation into methyl radicals. We use canonical transition state theory as discussed by Benson. ${ }^{25}$ This is not the most accurate theory available, but it serves to illustrate the contribution of hindered rotation without unnecessary complication. See Marcus $^{26}$ for a discussion of microcanonical methods applied to methyl radical recombination. In transition state theory, the rate constant for unimolecular dissociation $\left(k_{d}\right)$ can be expressed as

$$
k_{d}=(k T / h) K^{\ddagger},
$$

where $K^{\ddagger}$ is the equilibrium constant for formation of the activated complex with the reaction coordinate vibration factored out. An equilibrium constant can always be expressed as a ratio of partition functions. In the present case, this leads to

$$
K^{\ddagger}=\frac{q^{\ddagger}}{q^{r}}=g_{e} \frac{\left(q^{\mathrm{rot}} \Pi_{i=1}^{3 n-7} q_{i}^{\mathrm{vib}}\right)^{\ddagger}}{\left(q^{\mathrm{rot}} \Pi_{i=1}^{3 n-6} q_{i}^{\mathrm{vib}}\right)^{r}} \exp \left[\frac{-\Delta E}{k T}\right] .
$$

In Eq. (41), $\Delta E$ is the potential energy of the transition state relative to the reactant, $q^{\text {rot }}$ is the partition function for rigid rotation of the molecule as a whole, $q^{\text {vib }}$ is the vibrational partition function for each of the internal degrees of freedom, $r$ refers to the reactant molecule, and $\ddagger$ refers to the transition state. Since $K^{\ddagger}$ is the ratio of partition functions, this application serves to test the magnitude of the partition function. We assume that the transition state and equilibrium ethane are singly degenerate $\left(g_{e}=1\right)$ with $D_{3 d}$ symmetry $\left(\sigma^{\ddagger}=\sigma^{r}\right)$. This simplifies the equilibrium constant

$$
K^{\ddagger}=\left(\frac{I_{A}^{\ddagger} I_{B}^{\ddagger} I_{C}^{\ddagger}}{I_{A}^{r} I_{B}^{r} I_{C}^{r}}\right)^{1 / 2} \frac{\left(\Pi_{i=1}^{3 n-7} q_{i}^{\mathrm{vib}}\right)^{\ddagger}}{\left(\prod_{i=1}^{3 n-6} q_{i}^{\mathrm{vib}}\right)^{r}} \exp \left[\frac{-\Delta E}{k T}\right] .
$$

Here, $I_{A}, I_{B}$, and $I_{C}$ are the principle moments of inertia of the molecule as a whole.

We use a published parametrization of the potential energy surface along the minimum energy path for dissociation. ${ }^{27}$ It gives the vibrational frequencies, the barrier to internal rotation, and the molecular geometry as a function of the separation between carbon atoms. This distance is identified as the reaction coordinate. The transition state is the point along the reaction coordinate that minimizes $K^{\ddagger}$.

Figure 5 shows that using the HRDS function leads to slightly better agreement with the experimental results than using T91 over the entire temperature range covered. The largest difference between the approximations occurs when $\Theta \approx 2 r / 3$. At this point, ethane is intermediate between the harmonic oscillator and the free rotor limit and the ratio between HRDS and T91 is about 1.2. The transition state for ethane dissociation is "loose." This means that the carboncarbon bond is nearly broken and the hindering potential is very small at the transition state. In the free rotor limit, 


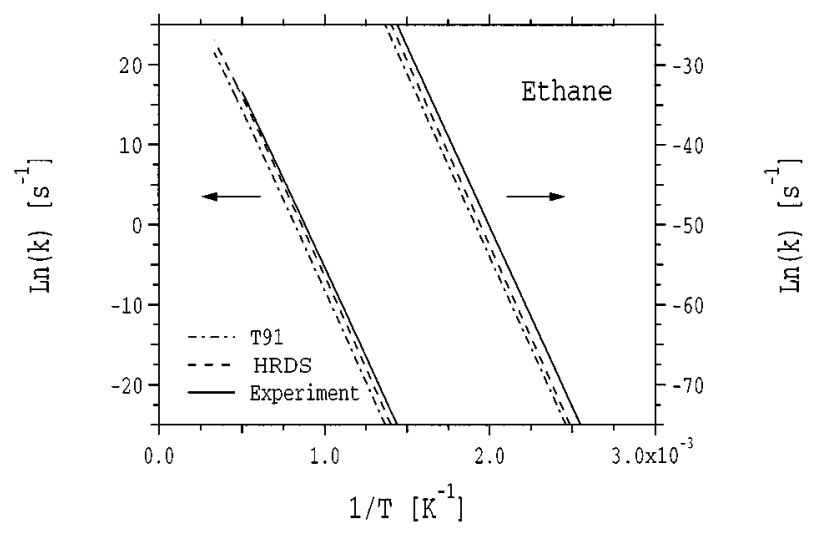

FIG. 5. High-pressure, unimolecular rate constant for dissociation of ethane into methyl radicals. The calculated values are based on Robertson's potential energy parametrization (Ref. 27) and two approximations for the hindered rotor. The experimental curve is a correlation from a critical analysis of kinetic and thermodynamic data (Ref. 28). Calculations based on the current work (HRDS) are closer to the experimental values than T91, but the maximum ratio between them is within the uncertainty of the experimental values. (The author estimates this uncertainty at a factor of 2 , which is \pm 0.7 on this scale.)

HRDS and T91 agree, so their ratio approaches one. Therefore, the largest difference between the estimates for reaction rates is about $20 \%$, which is well within the error bounds for kinetics experiments. Since the HRDS function is just as easy to use and more accurate, it can still be recommended over T91 even though kinetics experiments are unlikely to confirm one over the other.

\section{CONCLUSIONS}

We developed the HRDS interpolation function for readily estimating the partition function and thermodynamic functions for hindered rotors. The function is considerably easier to use than recalculation from first principles or interpolating from existing tables. ${ }^{1,2}$ Our approximant to the partition function (HRDS) is based entirely on the asymptotic behavior of the quantum-mechanical partition function at low temperature and the classical partition function at high temperature. There are no fitting parameters in this function. We have shown this function to be accurate in the temperature range intermediate between the harmonic oscillator and free rotor limits. We expect that this interpolation function will be useful in estimating thermodynamic and kinetic data for molecules and solids with hindered rotation about one axis.

\section{ACKNOWLEDGMENTS}

This research was funded by the NSF (CHE 95-22179 and ASC 92-17368). The facilities of the MSC are also sup- ported by grants from DOE-BCTR, Chevron Petroleum Technology, Asahi Chemial, Aramco, Owens Corning, Chevron Chemical Co., Asahi Glass, Chevron Research and Technology Co., BP Chemical, Hercules, Avery Dennison, and Beckman Institute.

${ }^{1}$ K. S. Pitzer, J. Chem. Phys. 5, 469 (1937).

${ }^{2}$ K. S. Pitzer and W. D. Gwinn, J. Chem. Phys. 10, 428 (1942).

${ }^{3}$ S. Mizushima, Structure of Molecules and Internal Rotation (Academic, New York, 1954).

${ }^{4}$ W. J. Orville-Thomas, Internal Rotation in Molecules (Wiley, London, 1974).

${ }^{5}$ D. G. Lister, J. N. Macdonald, and N. L. Owen, Internal Rotation and Inversion (Academic, London, 1978).

${ }^{6}$ E. B. Wilson, Jr., Adv. Chem. Phys. 2, 367 (1959).

${ }^{7}$ J. Dale, Tetrahedron 22, 3373 (1966).

${ }^{8}$ J. P. Lowe, Prog. Phys. Org. Chem. 6, 1 (1968).

${ }^{9}$ H. Förster and F. Vögtle, Agnew. Chem. 16, 429 (1977).

${ }^{10}$ D. A. Long, J. Mol. Struct. 126, 9 (1985).

${ }^{11}$ U. Berg and J. Sandström, Adv. Phys. Org. Chem. 25, 1 (1989).

${ }^{12}$ D. G. Truhlar, J. Comput. Chem. 12, 266 (1991).

${ }^{13}$ D. A. McQuarrie, Statistical Mechanics (Harper \& Row, New York, 1976), pp. 10, 58, 96-98, and 138-140.

${ }^{14}$ N. W. McLachlan, Theory and Application of Mathieu Functions (Dover, New York, 1964), pp. 10, 17, and 371. For convenience, we have used $-q=r^{2}$.

${ }^{15}$ Handbook of Mathematical Functions, edited by M. Abramowitz and I. A. Stegun (Dover, New York, 1965), pp. 374-379 and 722-726. This reference uses different, but equivalent notation for the Mathieu function. The following rules can be used to translate their notation to ours: $r \rightarrow m$, $a \rightarrow \alpha$, and $q \rightarrow-r^{2}$.

${ }^{16}$ H. H. Nielsen, Phys. Rev. 40, 445 (1932).

${ }^{17}$ R. V. Churchill and J. W. Brown, Complex Variables and Applications, 5th ed. (McGraw-Hill, New York, 1990), pp. 173-174.

${ }^{18}$ I. S. Gradshteyn and I. M. Ryzhik, Tables of Integrals, Series, and Products, 5th ed. (Academic, San Diego, 1994), pp. 354, 961, 968, 971, and 973.

${ }^{19}$ D. Zwillinger, Handbook of Differential Equations, 2nd ed. (Academic, Boston, 1992), pp. 223-230.

${ }^{20}$ The eigenvalues $(\alpha)$ were calculated using a program written by one of us (R.B.M.). The program uses the LAPACK routines for the matrix diagonalization. E. Anderson et al., LAPACK (SIAM, Philadelphia, 1992).

${ }^{21}$ W. H. Press, S. A. Teukolsky, W. T. Vetterling, and B. P. Flannery, Numerical Recipes in FORTRAN, 2nd ed. (Cambridge University Press, Cambridge, 1992), pp. 229-233.

${ }^{22}$ S. Wolfe, Acc. Chem. Res. 3, 102 (1970).

${ }^{23}$ J. Chao, R. C. Wilhoit, and B. J. Zwolinski, J. Phys. Chem. Ref. Data 2, 427 (1973).

${ }^{24}$ D. G. Friend, H. Ingham, and J. F. Ely, J. Phys. Chem. Ref. Data 20, 275 (1991).

${ }^{25}$ S. W. Benson, The Foundations of Chemical Kinetics (Krieger, Malabar, 1982), pp. 247-250.

${ }^{26}$ D. M. Wardlaw and R. A. Marcus, J. Phys. Chem. 90, 5383 (1986).

${ }^{27}$ S. H. Robertson, D. M. Wardlaw, and D. M. Hirst, J. Chem. Phys. 99, 7748 (1993).

${ }^{28}$ D. L. Baulch et al., J. Phys. Chem. Ref. Data 23, 847 (1994). 